\title{
Characterization of the CCD and CMOS cameras for grating-based phase-contrast tomography
}

\author{
Pavel Lytaev ${ }^{\mathrm{a}}$, Alexander Hipp ${ }^{\mathrm{a}}$, Lars Lottermoser ${ }^{\mathrm{a}}$, Julia Herzen ${ }^{\mathrm{d}}$, Imke Greving ${ }^{\text {a }}$, \\ Igor Khokhriakov $^{\mathrm{a}}$, Stephan Meyer-Loges ${ }^{\mathrm{b}}$, Jörn Plewka ${ }^{\mathrm{b}}$, Jörg Burmester ${ }^{\mathrm{b}}$, Michele Caselle ${ }^{\mathrm{c}}$, \\ Matthias Vogelgesang ${ }^{c}$, Suren Chilingaryan ${ }^{c}$, Andreas Kopmann ${ }^{c}$, Matthias Balzer ${ }^{c}$, \\ Andreas Schreyer ${ }^{\mathrm{a}}$, and Felix Beckmann ${ }^{\mathrm{a}}$ \\ ${ }^{a}$ Institute of Materials Research, Helmholtz-Zentrum Geesthacht, Max-Planck-Strasse 1, 21502 \\ Geesthacht, Germany; ${ }^{b}$ Central Technical Department, Electronics, Helmholtz-Zentrum Geesthacht, \\ Max-Planck-Strasse 1, 21502 Geesthacht; ${ }^{\mathrm{c}}$ Institute for Data Processing and Electronics, Karlsruhe \\ Institute of Technology, Hermann-v.Helmholtz-Platz 1, 76344 Eggenstein-Leopoldshafen, Germany. \\ ${ }^{\mathrm{d}}$ Department of Physics (E17), Technische Universität München, James-Franck-Strasse 1, 85748 \\ Garching, Germany.
}

\begin{abstract}
In this article we present the quantitative characterization of CCD and CMOS sensors which are used at the experiments for microtomography operated by HZG at PETRA III at DESY in Hamburg, Germany. A standard commercial CCD camera is compared to a camera based on a CMOS sensor. This CMOS camera is modified for grating-based differential phase-contrast tomography.

The main goal of the project is to quantify and to optimize the statistical parameters of this camera system. These key performance parameters such as readout noise, conversion gain and full-well capacity are used to define an optimized measurement for grating-based phase-contrast. First results will be shown.
\end{abstract}

Keywords: grating-based interferometry, phase-contrast tomography, UFO-project, CCD and CMOS sensors

\section{INTRODUCTION}

The Helmholtz-Zentrum Geesthacht (HZG) is operating microtomography stations using synchrotron radiation at the high brilliance third-generation synchrotron light source PETRA III at Deutsches Elektronen-Synchrotron (DESY) in Hamburg. To cover the full photon energy range from 7 to $150 \mathrm{keV}$ dedicated systems are energies and are installed at the Imaging Beam Line (IBL, P05) for 7 to $50 \mathrm{keV}$ and at the High Energy Material Science (HEMS, P07) for 35 to 150 $\mathrm{keV}$. Absorption contrast and phase-contrast techniques were developed and applied to samples in the fields of medicine, biology, and materials science.

One of the main advantages of using high brilliance synchrotron radiation is the ability to perform artifact free imaging methods. The low divergence of the monochromatic X-ray beam together with the high X-ray photon flux allow for measurements with well-defined condition. Therefore, quantitative investigations applying microtomography using absorption-contrast and phase-contrast can be performed showing not only a high spatial resolution but also a high density resolution [1-5]. Furthermore, the system can be optimized to perform fast imaging with a relatively high signal to noise ratio. This is particularly important in tomography techniques based on sets of correlated images. Especially, for tomography applying grating-based X-Ray Differential Phase-Contrast (DPC) a single projection is calculated from a set of images to resolve small refraction angles. An absorbing grating is used to scan an interference pattern, which is produced behind a periodic phase grating by means of the Talbot effect [6]. The first measurements of refraction with Xrays using a grating interferometer were realized early in this century at synchrotron sources $[7,8]$.

To build an optimized setup for DPC tomography for the beamlines IBL and HEMS at PETRA III the HZG in cooperation with the KIT are developing a camera based on an CMOS sensor and the ultrafast streaming camera 
platform (UFO-project) with programmable FPGA control unit $[9,10]$. Several camera prototypes have been tested with a tabletop setup including a visible light source. The data obtained in those test experiments were used to calculate some of the camera's key performance parameters such as readout noise, conversion gain and full-well capacity by using the Photon Transfer Curve method. To optimize the camera characteristic several modifications of the camera components were developed.

In this article we present the method and the results of the camera tests. Also a comparison of the camera characteristics with a commercial CCD camera used for absorption contrast at the stations for microtomography is presented. Moreover, first results of the current camera prototype performing a measurement of a single projection using grating-based DPC imaging of a mouse bone sample at our synchrotron station (PETRA III, P07, HEMS) are presented.

In the following subsection the photon transfer curve method is introduced in more details. In the "methods" section there are two subsections describing the camera development and the measurement procedure to obtain the photon transfer curve for the retriever of the statistical characteristics. In the following sections we present the results of the camera characteristic tests CMOS camera and the CCD camera. The last subsection is devoted to the first results obtaining a single projection with the setup for DPC using both camera systems.

\subsection{Photon transfer curve method}

Since two decades a test method of quantitative comparison of camera's key performance parameters has existed and is known as the Photon Transfer Curve (PTC) first described by J. R, Janesick [11]. The PTC characterization method is based on a theory where knowledge of a system's input and output signals are used to derive the characteristics of the system itself. In the simplest case the method it only requires the taking of pairs of image frames in a progressive time series of constant illumination and does not require complicated or specialized equipment, except a precisely controlled source of light which provides a flat (uniform) illumination field. The use of visible monochromatic light is important to remove the effects of color temperature and quantum efficiency variations of the used image device.

From a very basic measurement point of view we know that the camera itself is a system block with light as an input, and digital data as an output. Also due to the nature of photons themselves, the only noise introduced at the input is a shot noise, so we can predict exactly what that noise will be at a given illumination level. Furthermore we can assume that any difference between the noise at the input and the noise at the output must have been caused by the camera electronics.

In summary, a typical noise profile observed at the output of a digital camera contains three distinct noise regions: readout noise, shot noise, and fixed pattern noise (Fig. 1). And as described in [12] the total noise in Analog/Digital Units (ADU) for an exposure is given by:

$$
\sigma_{\text {total }}=\left(\sigma_{\text {read }}^{2}+\left((S / k)^{0.5}\right)^{2}+(F P N \times S)^{2}\right)^{0.5}
$$

where the total noise is obtained by taking the square root of the quadrature sum of the individual noise components:

- $\sigma_{\text {read }}$ is the readout noise which means the random noise associated with the camera output amplifier and its (external) signal processing electronics. This is also referred to as the noise floor of the camera, and represents the baseline noise in total darkness. Thus, in a good case the readout noise is represented by a horizontal line at the very beginning of the PTC.

- $(S / k)^{0.5}$ is the shot noise in ADU arising from the photon flux; $S$ is the signal (number of counts in ADU) and $k$ is the conversion gain in electrons/ADU. (Suppose the total number of photons per pixel in an exposure is $N$ and assume that each photon results in one electron. Then the number of electrons is $N$, and the shot noise is the square root of $N$ i.e. $\operatorname{sqrt}(N)$. When the number of electrons is converted to ADU, the signal in ADU becomes $S$ $=N / k$ but the noise is now $\operatorname{sqrt}(N) / k$, because the 'width' of the Gaussian shot noise distribution is scaled by the same factor as the mean value. Since $N=S \times k$, the noise in ADU is $s q r t(S / k))$. Thus, the shot noise profile becomes a straight line with a slope of half on the $\log -\log$ curve $\left(\log X^{\wedge} 0.5=0.5 \log X\right)$.

- $F P N \times S$ is the fixed pattern noise arising from non-uniformity of response of the CCD chip, and it becomes dominant at relatively high levels of illumination. Suppose we consider two neighboring pixels where one has 
half the sensitivity of the other. If one exposure resulted in 100 counts in one pixel and 200 in the other, an exposure of twice the length would give 200 and 400 counts respectively. So the fixed pattern noise scales linearly with the signal and its profile becomes a straight line with a slope of one.

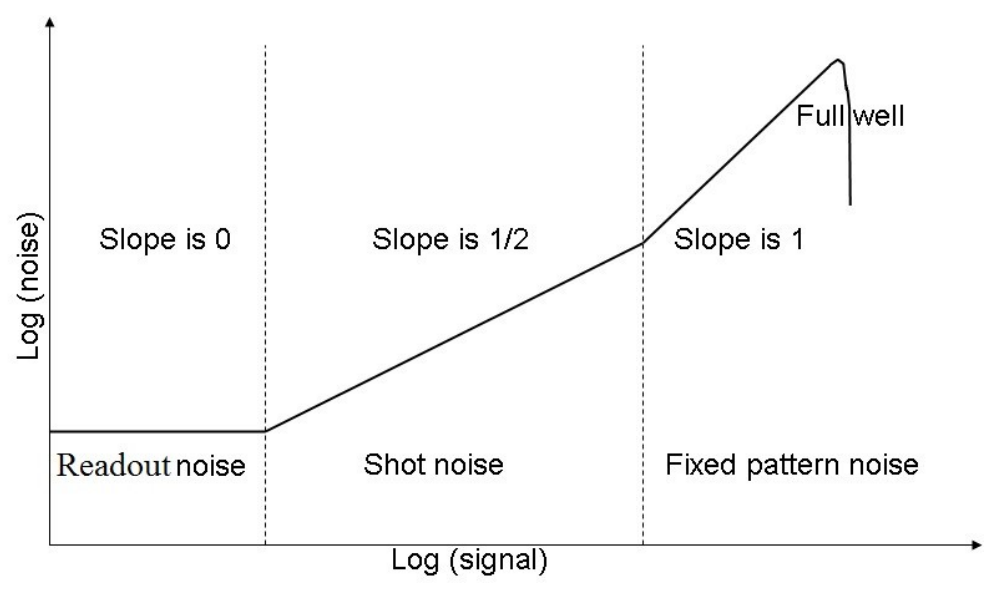

Figure 1. The photon transfer curve is presented schematically with three distinct regions dominated by different kind of noise.

Recall again, the PTC measurement requires at least a pair of image frames at the given illumination level. The average number of counts for each pair gives the mean signal value, and the average standard deviation for each pair gives the total noise. The fixed pattern noise can be removed by the differencing process of the pair. Subtracting the two illuminated fields eliminates fixed pattern variations since they are present in both images.

As illumination levels are increased, the individual sensor pixels eventually become unable to hold any additional charge without "spilling-over" into neighboring wells. At this point on the noise curve, output noise abruptly drops because charge sharing between neighboring pixels averages the signal and suppresses random noise. At the point where the PTC peaks, the sensor has reached a so called full-well limit. In our case the fixed pattern noise is eliminated as we perform the calculation for each pixel element. Therefore, the shot noise region is extended and the PTC continues along with a slope of $1 / 2$ until full well occurs.

As described in [13], once the noise at each illumination level is calculated, a PTC is generated by plotting the camera's output average standard deviation (rms of noise) vs. average signal level on a Log-Log curve. Then readout noise is directly available from PTC by recording the noise level at zero illumination. The full-well capacity parameter is the signal level at the point where the PTC drops. The conversion gain G of camera systems is typically expressed in $\mathrm{ADU} / \mathrm{e}-$, that is, the number of $\mathrm{A} / \mathrm{D}$ units per signal electron. To obtain this gain, we note that for an increase in illumination of $\mathrm{X}$, the camera's average signal level will change by GX. In contrast, the same increase in illumination level will cause the noise variance, to change by $G^{2} X$, then: $\log \left(G^{2} X\right)=\log G X+\log G$. That means that on the logarithmic plot an intersection between the line interpolating the PTC part with slope of half and the $\mathrm{x}$-axis gives the Log G. Thus, by plotting the photon transfer curve in logarithmic scale we can easily find the camera's key performance parameters by graphical means.

\section{METHODS}

\subsection{The camera development}

In accordance with our primary goal - to build up an optimized detector for grating-based DPC imaging - the first prototype of a smart camera was developed by engineers of Karlsruhe Institute of Technology as part within the UFOproject $[9,10]$. The prototype consisting of an CMOS sensor, focusing optics and, a smart electronic platform is shown in Figure 2, A. 


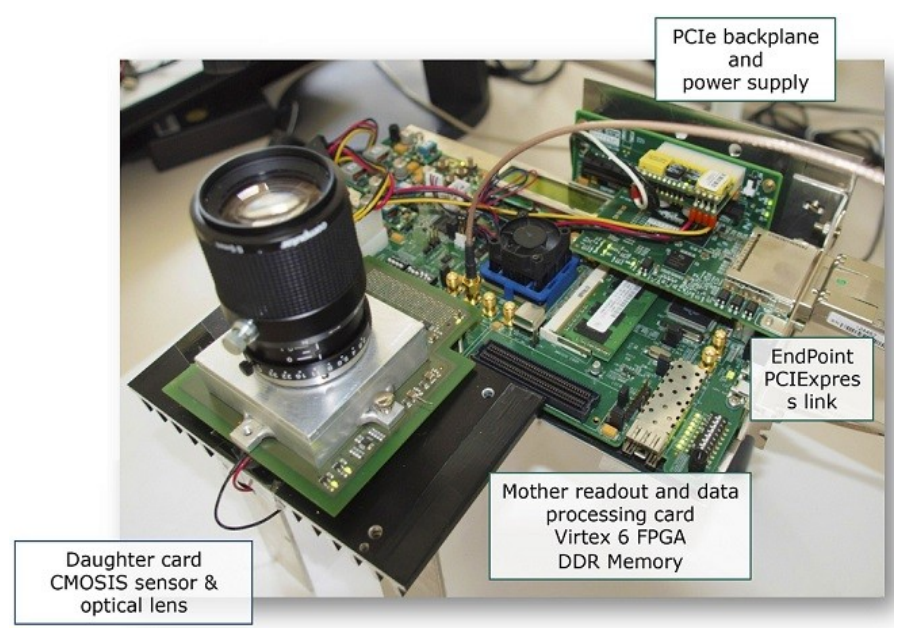

A

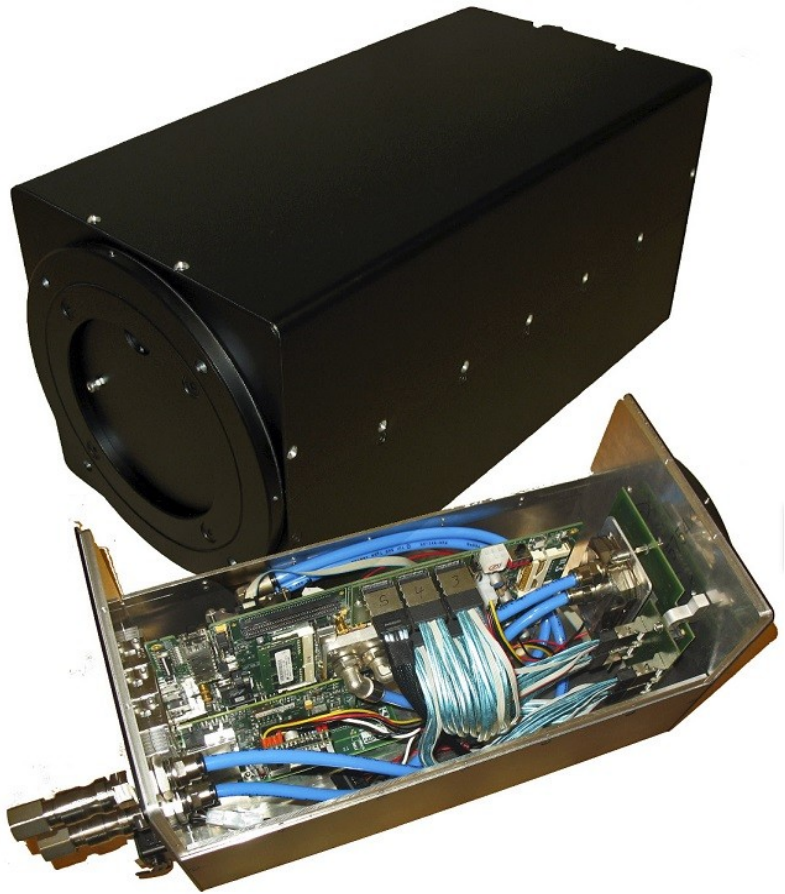

B

Figure 2. A - Prototype of the camera system consisting of an CMOS sensor as part of the UFO-project. B - smart scientific camera based on the same platform, but with modifications e.g. cooling, housing, enhanced power supply developed within this project.

In more details, the name and parameters of the camera sensor are as follows: 12 bit CMOS Image Sensor, CMV20002E5M1PP monochrome, 2048x1088 array size, 5.5 um pixel size. Concerning throughput it is a moderate frame rate speed image-sensor with a bandwidth of $7.7 \mathrm{~Gb} / \mathrm{s}$. The electronics of the camera system contains an FPGA-based programmable platform and GPU-based evaluation unit (Fig. 3).

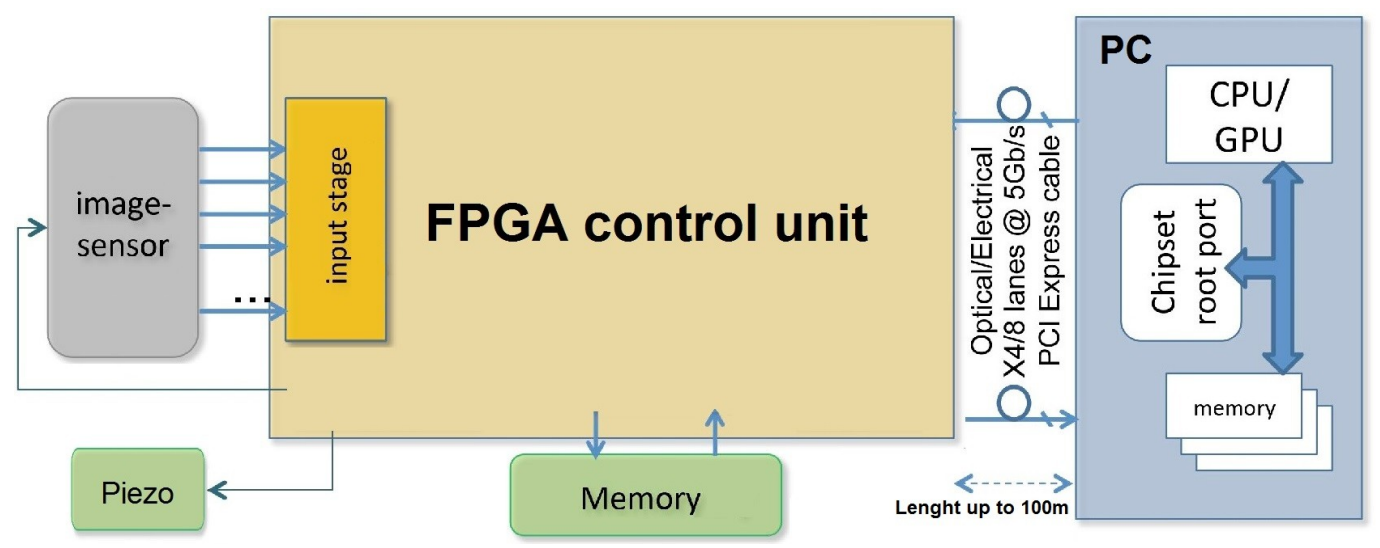

Figure 3. Schematic of the smart camera platform architecture based on the UFO-project $[9,10]$.

In order to install the camera at the microtomography stations at PETRA III, several modifications of the prototype were done by the electronic department in Geesthacht. Several changes in the geometry of the constituent elements of the camera were done to integrate the cooling system for the sensor and the enhanced power supply in a housing fitting into 
the detector tower at the beamline. The result of this work is shown in Figure 2, B. The main features of the present system are:

- $\quad$ Enhanced power supply (http://shop.rohde-schwarz.com/oeat/hamegr-hmp2030.html)

- $\quad$ Peltier cooling system of sensor controlled by FPGA

- $\quad$ Full streaming readout e.g. using PCIe (Linux drivers, Tango device server)

- Fully configurable camera (exposure time, dynamic range, analog and digital pixel features)

- $\quad$ Region-of-interest readout strategy using self-event trigger information

- $\quad$ Easily extendable to any available CMOS image sensor

- Continuous data acquisition at full speed

\subsection{Data acquisition and treatment}

To characterize and optimize the CMOS camera system we measure the photon transfer curve with a tabletop setup. It consists of a dark box containing the holder for the sensor with its electronics and an illumination box as a constant light source. To provide a flat (uniform) illumination field a High Power LED (color cyan, $492 \mathrm{~nm}$ ) was connected to an integrating Ulbricht sphere with an inner white surface and a circular aperture. The camera sensor was placed in front of and next to the output of the illumination box (Fig. 4). The electronic used for adjustment of the setup and for the experimental control were based on Beckhoff PLC via Modbus TCP. The scripting of the measurement were done using batch programming in Linux.

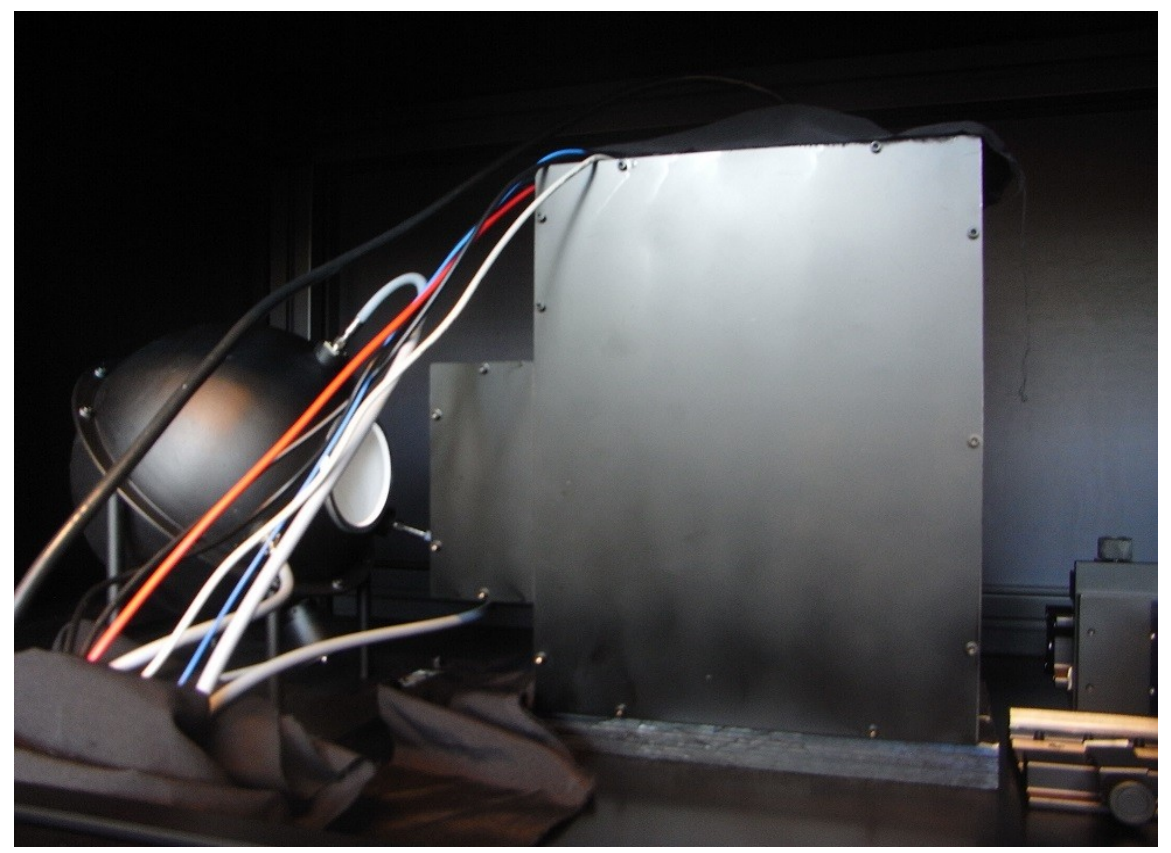

Figure 4. Picture of the setup for PTC measurements. On the left side of the picture the integrating sphere is shown. As a light source an LED is connected to the sphere. On the right side the box containing the camera with its electronics is shown.

To measure the readout noise and also to make a first estimation of the camera statistics we obtained a set of so called "dark current" image frames, i.e. we varied the exposure time of the sensor in total darkness. The set is series of groups of images, each group is made for the same exposure time, but the exposure time is varied from group to group. Each group contains several picture frames. By plotting for a given pixel the mean signal value of each group versus the exposure time we normally assume a horizontal linear behavior. If the variance within the groups is too high (in the same 
range as the signal value or more) we observe only a noisy structure on the plot and the parameters for the $\mathrm{AD}$ conversion have to be modified. If we receive a linear behavior with respect to the exposure time showing a positive slope, the cooling of the sensor has to be enhanced. Thus, having a normal case we can estimate the readout noise by reading the intercept of the corresponding line on the y-axis.

For the measurement of the full PTC sets of images at varying exposure times are measured. The range for the exposure time is selected starting from mean values in the range of the dark current up to a fully exposed camera sensor. For each set the mean value and the variance is calculated. For data evaluation we used the software IDL 8.2 with its own programming language (Interactive Data Language) [14]. For the pairs showing the linear behavior in the "shot noise" region of the PTC (s. Fig. 1) the conversion gain is defined by the linear regression line. The PTC then can be plotted showing the readout noise, conversion gain, and the full-well capacity for each sensor element (Pixel). To quantify the behavior of the full sensor, 2-dim. images of these statistical parameters are created.

\section{EXPERIMENTAL RESULTS AND DISCUSSION}

\subsection{1 Characterization of in house camera with CMOS sensor}

As described in "Methods" we have performed the characterization measurement of the camera system described in paragraph 2. For the "dark current" test we took four image frames at the given exposure time starting from zero with equidistant steps of $100 \mathrm{~ms}$ up to $6800 \mathrm{~ms}$. The result for a single pixel (pix 0550_0913) is presented in Figure 5A as a plot of the mean value for each group of four images versus exposure time. The linear behavior with a slope of slightly above zero of the camera sensor can be seen. At the exposure time of $4500 \mathrm{~ms}$ a slight jump down to the original value at 0 exposure time can be detected, which have been caused by cooling the sensor down to originally set temperature of -10 $\operatorname{deg}$ C. It is also clear that overall dispersion is in a range of $20 / 300$ i.e. less than $6.7 \%$ and overall average mean value is about 290 counts. So, since our camera is operated in 12 bit mode and therefore, the maximum possible value is 4048 , we assume that the dark noise level is reasonable enough for the subsequent measurements.
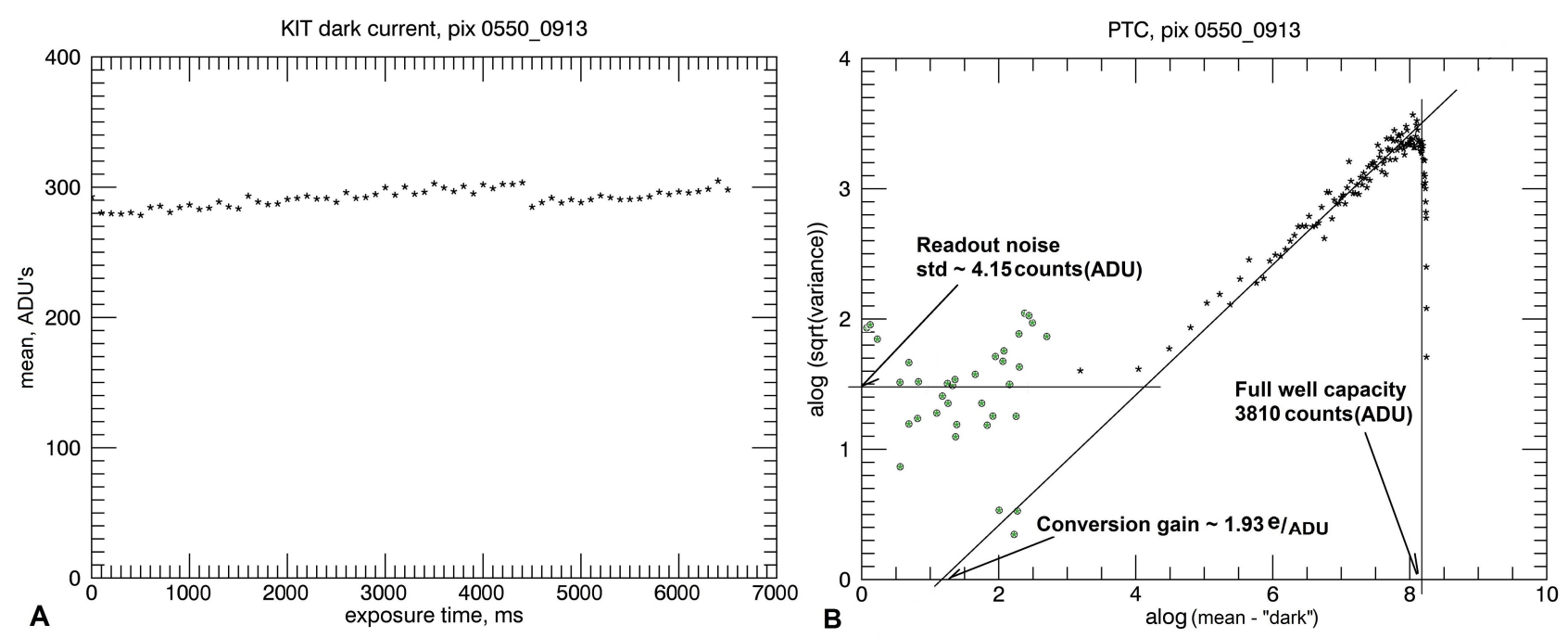

Figure 5. Results of the in house camera (CMOS) for the single pixel 550x913. A - plot of the mean values of photon counts versus exposure time, measured without using the light source (dark current test). B - the PTC with the key performance camera's parameters indicated. The green rounded stars are assigned for the point of the measurement for the "dark current".

The next step is the actual PTC measurement. This time we have 100 single images in each group at the given exposure time. Starting from zero with a step of $100 \mathrm{~ms}$ the exposure time goes up to 12 seconds in order to cover the sensor's full-well limit for sure. We used the IDL software to calculate, at first, the mean values and variances for every group of 100 pictures. At second, we made the corresponding 3D arrays with the full camera sensor ( $2048 \mathrm{x} 1088$ pixels) as the first two dimensions and all the mean values and variances including "dark points" as the third dimension. For a given pixel we assigned to a variable $\mathrm{X}$ the natural logarithm of difference between the "means array" value at a random point 
and its value at the first point (dark current). The variable $\mathrm{Y}$ is a natural logarithm of the "variances array" value at a random point, for the same pixel of course. By plotting Y versus X we obtain the PTC itself which are represented in Figure 5, B.

By fitting the first "dark current" points by a horizontal line and intersecting this line with the Y-axis we can figure out the value of natural logarithm of the readout noise. Also by finding the maximum Y-value at the right we calculate the full-well capacity parameter as the logarithm at the corresponding X-point. And finally, by fitting the points between the full-well limit and the first points with low-exposed light (in fact 30 points are used before $\mathrm{Y}$ reaches its maximum) by the line with slope of $1 / 2$ and intersecting this line with the $\mathrm{X}$-axis we obtain the corresponding logarithm of the conversion gain. All the points, fitting lines and parameters values are also represented in the same plot in Figure 5, B.

To have an impression of the PTC behavior not only for a single pixel but for the whole camera sensor, the distributions of known key parameters at all the matrix pixels is shown in Figure 6. The characteristic of CMOS chip banded structure is clearly visible [15]. As a positive result we can conclude that for all the three parameters the grayscales limits are quite close to each other, i.e. almost all the matrix pixels behave similar.

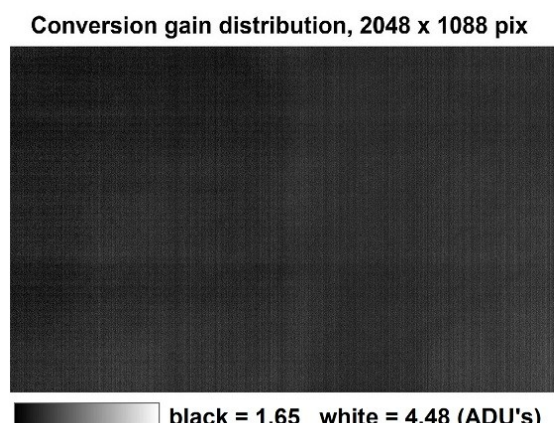

A

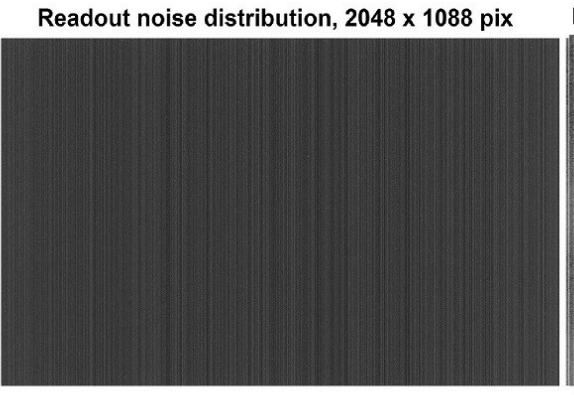

B
Full well capacity $\left(\mathrm{x}_{\max }\right)$ distribution, $2048 \times 1088 \mathrm{pix}$

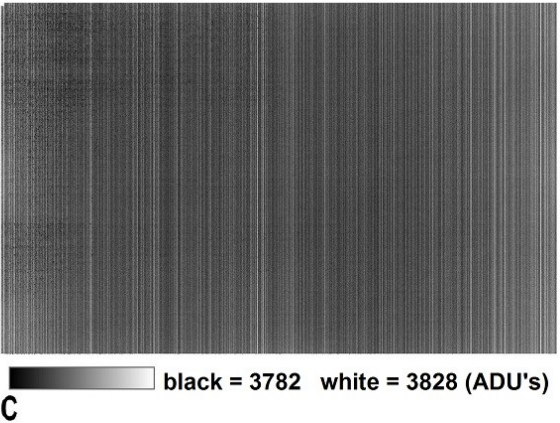

Figure 6. Distribution of the single parameter for the camera sensor in grayscale. A - conversion gain distribution; $\mathrm{B}$ - readout noise distribution; $\mathrm{C}$ - full-well capacity parameter distribution.

\subsection{Characterization of the commercial CCD camera}

To make a comparison of our results with some sort of standard camera we have also performed a similar PTC measurement with another camera system based on a CCD chip. The camera used is a commercial 14 bit CCD, SC09000M-NS-LCDD, KAF09000 monochrome, Digital Imaging System, 3056x3056 array size, 12 um pixel size. This camera is usually used for the high resolution imaging at the beamlines IBL and HEMS at PETRA III. The camera has two operational modes: one slow $1 \mathrm{MHz}$ mode and relatively fast $8 \mathrm{MHZ}$ mode. As we are normally using the fast mode we have performed the PTC measurement in this mode.

Since the storage ring PETRA III has been shut down on February 2014 we installed a similar testing setup as the described paragraph 2 at the beamline HEMS. The black camera tower you can see at the picture in Figure 8, and the light source was placed right in front of its input.

During the measurement we collected 25 single image frames at each illumination level for the "dark current" test and 100 image frames per exposure for the PTC test with illumination. Starting with $50 \mathrm{~ms}$ of exposure time due to the technical limitation, the illumination was increased by varying the aperture from 0,1 to 1.0 of its full size with step of 0.1 . Furthermore, the exposure time was increased up to $860 \mathrm{~ms}$ with step of $10 \mathrm{~ms}$. The sensor temperature was set to $-15 \operatorname{deg} \mathrm{C}$.

As a result the photon transfer curve for a single pixel is represented in Figure 7. On the plot we can see almost the same typical behavior of the PTC as for our in house camera (CMOS) presented in Figure 5B. Due to the different electronic design of the CCD system significant differences can be seen in the full-well capacity and the readout noise values. Also a more linear and horizontal behavior of the "dark current" points can be identified, which can be caused by better temperature stability of the system. 


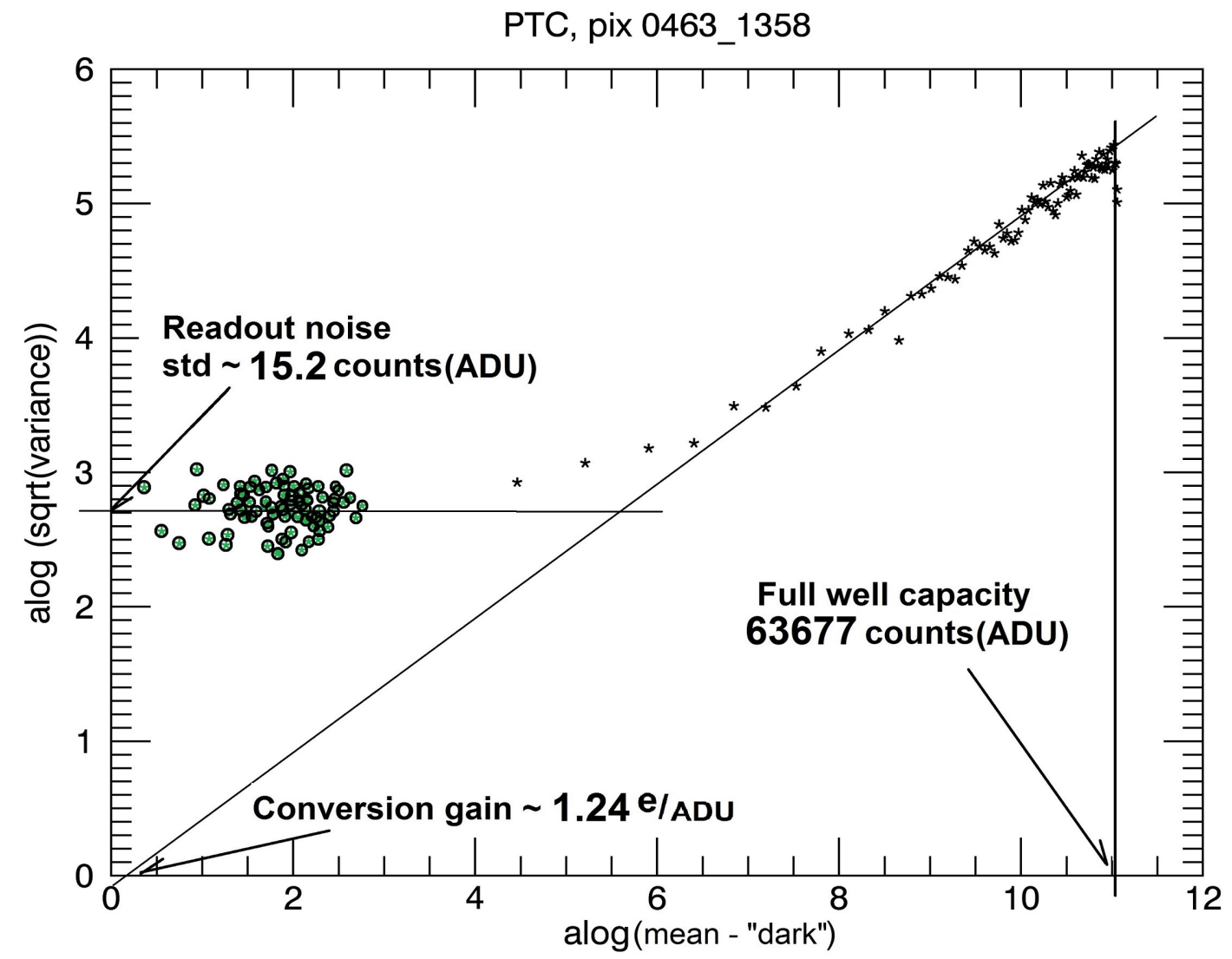

Figure 7. The PTC for the single pixel $(463 \times 1858)$ of the CCD camera with its key parameters indicated. The green rounded stars are assigned for the "dark current".

\subsection{Grating-based DPC experiment @ HEMS}

To test the in house camera based on the CMOS sensor in a real experiment, installed the DPC imaging setup at the HEMS beamline in January 2014. The setup for DPC and the internal installation of the camera tower is shown in Figure 8. The camera tower covers slot for two different camera systems. Both cameras tested in this paper were installed and the mounting can be seen Figure 8, B. We performed DPC projection measurements using different scan parameters. We varied the number of phase steps, the number of periods scanned and the exposure time. For both camera systems phase projection are measured and calculated using the same optical magnification of the detector system. As the sensor area of the CMOS and CCD system is different the field of view of the systems differs.

For the presented DPC setup we used a grating interferometer consisting of $\pi$-shifting phase grating G1, made of nickel with a period of $4.8 \mathrm{um}$ and height of the bars of $16 \mathrm{um}$, and the absorption gold grating G2 with a period of $2.4 \mathrm{um}$ and height of the bars of $120 \mathrm{um}$. The distance between the G1 and G2 was approximately $21 \mathrm{~cm}$. For the stepping process we used a piezo drive. As the test object we used a well-prepared sample of a mouse bone. The sample was kept in $70 \%$ ethanol in a plastic tube of about $7 \mathrm{~mm}$ thick. The x-ray beam energy was set to $35 \mathrm{keV}$. At this energy there was no need to use a water bath for preventing artifacts at the air-plastic boundaries. The setup, experimental procedure and reconstruction process are described in more details in [16].

As a result we present two single phase projections corresponding to each camera (Fig. 9). The phase shift caused by the sample structure is scaled in grayscale. The measurement was done using the exposure time of $550 \mathrm{~ms}$ for the CCD camera, and $100 \mathrm{~ms}$ for the CMOS camera. In both cases we made 10 phase steps over one period by moving the G1 using a piezo. Looking at the pictures we clearly can see that the image quality and the resulting contrast are comparable for both pictures. The field of view (FOV) is smaller for the CMOS camera, naturally due to its smaller matrix size. We can conclude that with the CMOS camera system we are able to perform the imaging with a comparable quality. 


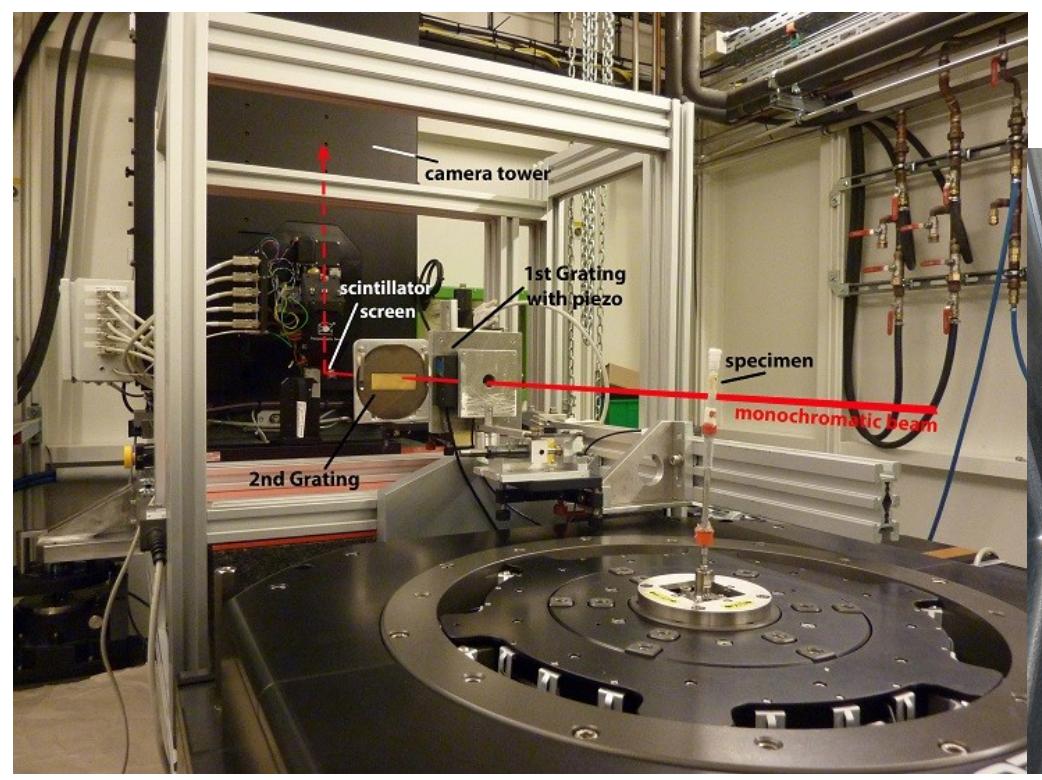

A

Figure 8. A - DPC setup at HEMS beamline. B - CCD and CMOS cameras inside the camera tower.

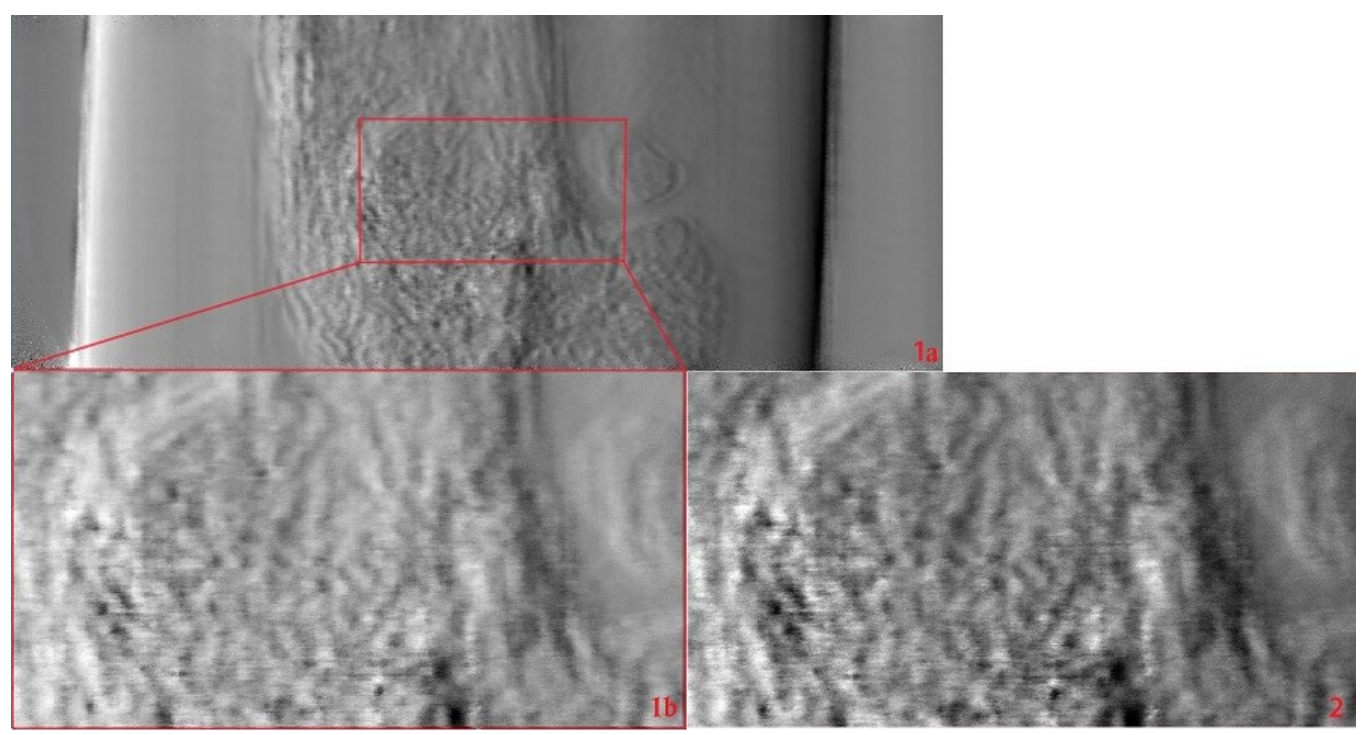

Figure 9. Results showing a single projection of the mouse bone sample. 1a - reconstructed phase projection obtained with the use of the CCD camera, FOV is $7.3 \times 2.9 \mathrm{~mm}$. $1 \mathrm{~b}$ - the part of the same projection to compare with the CMOS camera results, FOV is $2.3 \times 1.2 \mathrm{~mm}^{2} .2$ - reconstructed phase projection obtained with the use of the CMOS camera, FOV is $2.3 \times 1.2 \mathrm{~mm}$. In both the pictures phase shifts of the sample structure are represented in grayscale, where white is -2.5 , and black is 2.5 .

\section{SUMMARY AND OUTLOOK}

In this article we have presented the results for the characterization of different camera systems by measuring the photon transfer curve. One of those cameras was a prototype of a smart CMOS camera system, which is planned to be used for grating-based X-ray DPC imaging. The key performance characteristics of this camera have been compared to the corresponding parameters of a standard commercial CCD camera. The present system is used to test, optimize and quantify the statistical parameters of the 2-dim. sensors used for imaging at the beamlines IBL and HEMS at PETRA III 
Furthermore, we have presented the first results of experimental tests using the cameras with the setup for grating-based DPC projection imaging for one test specimen. During this experiment not only several projections, but also several large datasets with different scan parameters are obtained. These datasets together with the knowledge of the statistical parameters of the used sensors will be used to define optimized measurements for DPC with respect for scan time, and the statistical quality for the projection of the phase, absorption, and dark field.

\section{ACKNOWLEDGMENTS}

We would like to thank Katharina Jähn, PhD from Experimental Trauma Surgery University Medical Center HamburgEppendorf for providing us with the nice mouse bone sample to investigate. For providing the gratings for the DPC setup we thank the IMT Department of the KIT, Karlsruhe, Germany, within the Virtual Institute of New X ray Analytic Methods in Material Science (VI-NXMM).

We also want to thank all the technical staff of HZG at the DESY outstation, and especially Hilmar Burmester, Rene Kirchhof and Thomas Dose for their responsiveness and timely assistance in resolving related technical problems, which often occurred during the experiment.

The project is a part of the Helmholtz Detector Platform [17].

\section{REFERENCES}

[1] F. Beckmann, J. Herzen, A. Haibel, B. Müller, A. Schreyer, "High density resolution in synchrotron-radiation-based attenuation-contrast microtomography," Proc. SPIE 7078-1D, (2008).

[2] Herzen, J. et al., "X-ray grating interferometer for imaging at a second-generation synchrotron radiation source," Proc. SPIE 7804-1, (2010).

[3] Herzen, J., Donath, T., Beckmann, F., Ogurreck, M., David, C., Mohr, J., Pfeiffer, F. \& Schreyer, A., "X-ray grating interferometer for materials-science imaging at a low-coherent wiggler source," Review of Scientific Instruments Vol. 82, p. 113 (2011).

[4] Herzen, J., "A grating interferometer for materials science imaging at a second-generation synchrotron radiation source," PhD thesis, Department Physik der Universität Hamburg, pp. 13, 64-67, 75 (2010).

[5] Donath, T., "Quantitative X-ray Microtomography with Synchrotron Radiation," PhD thesis, Fachbereich Physik der Universität Hamburg, pp. 2-10, 46-47 (2006).

[6] Weitkamp, T. et al., "X-ray phase imaging with a grating interferometer," Optic. Express 13 6296, (2005).

[7] David, C., Nöhammer, B., Solak, H. H., and Ziegler, E., "Differential x-ray phase contrast imaging using a shearing interferometer," Appl. Phys. Lett. 81 3287, (2002).

[8] A. Momose et al., "Demonstration of X-Ray Talbot Interferometry," Jpn. J. Appl. Phys. 42 L866, (2003).

[9] Ultra-Fast X-Ray Imaging (UFO), < $\underline{\text { http://ufo.kit.edu }>\text {. }}$

[10] M. Caselle, S. Chilingaryan, A. Herdt, A. Kopmann, U. Stevanovic, M. Vogelgesang, M. Balzer, M. Weber, "Ultra-fast streaming camera platform for scientific applications," IEEE TNS Vol. 60, 5 (2013), 3669-3677

[11] Janesick, J. R., "CCD characterization using the photon transfer technique" in Prettyjohns, K. and E.Derenlak, eds., [Solid State Imaging Arrays], Proc. SPIE vol. 570, pp. 7-19 (1985).

[12] Janesick, J. R., [Photon Transfer DN to $\lambda$ ], ISBN 978-0-8194-6722-5, SPIE Press, p. 34 (2007).

[13] Gardner, D., "Characterizing digital cameras with the photon transfer curve," Summit Imaging, $<$ http://www.couriertronics.com/docs/notes/cameras_application_notes/Photon_Transfer_Curve_Charactrization_M ethod.pdf>, Accessed: (26 June 2012).

[14] IDL software description at the official website, <http://www.exelisvis.com/ProductsServices/IDL.aspx>.

[15] Holst, G. C. and Lomheim, T. S., [CMOS/CCD Sensors and Camera Systems], SPIE Press, pp. $94-99$ (2007).

[16] Hipp A., Lytaev, P. et al., "X-ray phase-contrast imaging at PETRA III," SPIE Optics + Photonics Proceedings, Paper No. 9212-5, (2014).

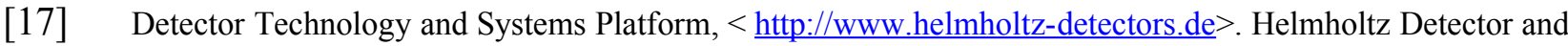
Systems Platform (DTS). 
